\title{
Lengthened Predelivery Stay and Antepartum Complications in Women with Depressive Symptoms During Pregnancy
}

\author{
Christie Lancaster Palladino, M.D., M.Sc., ${ }^{1,2,3}$ Heather A. Flynn, Ph.D., ${ }^{4}$ Caroline Richardson, M.D.,${ }^{5,6,7}$ \\ Sheila M. Marcus, M.D., Timothy R. B. Johnson, M.D., ${ }^{3}$ and Matthew M. Davis, M.D., MAPP ${ }^{8,9,10}$
}

\begin{abstract}
Background: It is crucial to understand the timing and mechanisms behind depression's effect on peripartum stay because attempts to intervene will vary based on the time period involved. We designed this study to compare predelivery and postdelivery length of stay in women with and without elevated depressive symptoms during pregnancy.

Methods: This study involved secondary data analysis of a larger study exploring antepartum depression. Each subject completed the Center for Epidemiological Studies Depression Scale (CES-D) during pregnancy at a mean of 25.8 weeks' gestation. We used time-stamped data to compare total peripartum, predelivery, and postdelivery lengths of stay in women with and without elevated depressive symptoms during pregnancy. In addition, we used a Cox proportional hazards regression model to evaluate potential mechanisms for depression's effect on length of stay.

Results: The study sample included 802 pregnant women. Overall, $18 \%$ of study subjects scored $\geq 16$ on the CES-D. Bivariate analyses demonstrated a significant association between elevated depressive symptoms and longer predelivery stays (time from admission to delivery). Interaction analyses demonstrated a significant interaction effect between depressive symptoms and parity, such that depressive symptoms were significantly associated with predelivery length of stay in multiparas but not so in primiparous subjects. In a multivariate model of multiparous subjects, depression's effect on length of stay was partially influenced by sociodemographic confounders but remained significant until antepartum complications were added to the model. Conclusions: Depressive symptoms during pregnancy are significantly associated with a subsequent increase in predelivery length of stay, and this association is mediated in part by antepartum complications, even after controlling for sociodemographic factors. These longer hospital stays can present significant burdens to the patient, her family, and the healthcare system. Future studies should evaluate whether interventions for depression during pregnancy can impact this relationship among depressive symptoms during pregnancy, antepartum complications, and extensive predelivery hospitalizations.
\end{abstract}

\section{Introduction}

D EPRESSION IS ONE OF THE MOST common complications in pregnancy, affecting approximately $12.7 \%$ of pregnant women. ${ }^{1}$ We hypothesized that depression during pregnancy may affect maternal length of stay at delivery (hereafter called "peripartum stay") because depression has been linked to length of stay outcomes in other clinical sam- ples, ${ }^{2}$ including hip fracture patients, ${ }^{3}$ general hospital inpatients, ${ }^{4,5}$ patients with chronic obstructive pulmonary disease (COPD), ${ }^{6}$ and coronary bypass patients. ${ }^{7}$ Our previous research has shown that depression during pregnancy is significantly related to a longer maternal stay at delivery and a $10 \%$ increase in hospital charges. ${ }^{8}$ With over 4.3 million births in the United States, ${ }^{9}$ this depression-related increase in peripartum stay equates to $>130,000$ excess hospital days and

\footnotetext{
${ }^{1}$ Department of Obstetrics and Gynecology and ${ }^{2}$ Education Discovery Institute, Georgia Health Sciences University, Augusta, Georgia.

${ }^{3}$ Department of Obstetrics and Gynecology, ${ }^{4}$ Department of Psychiatry-Women's Mood Disorders Program, ${ }^{5}$ Department of Family Medicine, ${ }^{6}$ VA Health Services Research and Development, ${ }^{10} \mathrm{Child}$ Health Evaluation and Research (CHEAR) Unit-Division of General Pediatrics, ${ }^{8}$ Gerald R. Ford School of Public Policy, and ${ }^{9}$ Division of General Internal Medicine, University of Michigan, Ann Arbor, Michigan.

${ }^{7}$ Veterans Affairs Medical Center, Ann Arbor, Michigan.
} 
a potential savings of almost 300 million dollars. We have yet to determine, however, whether depression's effect on length of stay is an antepartum (before labor), intrapartum (during labor), or postpartum (postdelivery) phenomenon because peripartum stay is usually recorded in aggregate, from the time of admission to the time of postpartum discharge.

Understanding the timing and mechanisms behind depression's effect on peripartum stay is crucial because attempts to intervene will vary based on the time period involved. For example, if anxious symptoms in pregnant women with depression are leading to an increased rate of labor induction, this would require a very different approach than if postpartum breastfeeding difficulties in women with depression were to explain depression's effect on peripartum length of stay.

We hypothesized that depression may affect the peripartum stay through several potential mechanisms (Appendix, supplemental material available online at www .liebertonline.com). For example, depression is linked to adverse obstetric outcomes, including preterm delivery and preeclampsia, $^{10-12}$ and, therefore, could lengthen maternal stay, particularly antepartum stays, by increasing the frequency of such complications. We also hypothesized that depressed mothers taking antidepressants could stay longer postpartum because of lengthened neonatal observations, as use of selective serotonin reuptake inhibitors (SSRIs) is associated with a neonatal withdrawal syndrome. ${ }^{13-17}$ Finally, depression may not independently influence peripartum stay, but it could appear to do so through an association with socioeconomic status (SES) or substance abuse. Other variables shown to be related to peripartum stay include obstetric factors (e.g., parity, mode of delivery, and complications of pregnancy), neonatal factors (e.g., birth weight, gestational age, and neonatal intensive care unit [NICU] admission), and sociodemographic factors (e.g., race/ethnicity, insurance status, and maternal age). ${ }^{18-27}$

The objective of this study was to compare predelivery length of stay (the time from admission to the obstetrics unit to the time of delivery) and postdelivery length of stay (the time from delivery to the time of postpartum discharge) in women with and without elevated depressive symptoms during pregnancy. Our previous study examining the question of maternal depression and peripartum length of stay was limited by the lack of detailed time of day information for critical events in the perinatal sequence, including time of admission, time of delivery, and time of discharge. ${ }^{8}$ Therefore, this study seeks to expand on previous research using detailed timestamped data from a large sample of perinatal admissions. In addition, we aimed to explore potential mechanisms by which depressive symptoms may impact maternal length of stay.

\section{Materials and Methods}

\section{Recruitment and study sample}

This study involved secondary data analysis of the Health and Pregnancy Project (HPP), a cohort study examining general health behaviors, substance abuse, and depression in pregnancy conducted from 1999 through 2003 by two of the investigators (S.M.M. and H.A.F.) (Fig. 1). A convenience sample of pregnant women was recruited from a group of obstetrics clinics throughout southeastern Michigan. Pregnant women were approached by research staff while waiting for a prenatal visit and were asked to complete a selfadministered survey. Comprehensive written consent was obtained from each study participant. The University of Michigan Medical School Institutional Review Board approved all procedures for the HPP and for this study.

Of the 1479 women approached, approximately 90\% agreed to complete the screening survey $(n=1331$ women screened). Women who chose not to participate refused further contact with the research assistant; therefore, it was not possible to collect information on their characteristics. In addition, we excluded 449 women who were patients in outside health systems, women with fetal deaths in utero $(n=6)$, and women who delivered at $<20$ weeks gestational age $(n=9)$. This yielded a sample size of 867 eligible women who delivered in our university health system, where time-stamped data for critical perinatal events were available (Fig. 2). Women were recruited in all three trimesters of pregnancy but at a mean of 25.8 weeks gestation (standard deviation [SD] 9.6, median 27, interquartile range [IQR] 18-34).

Of the 867 women in the study sample, 10 women were missing time-stamped data for admission to the obstetrics unit. Women with missing admission data did not differ by sociodemographic characteristics, induction rates, obstetric complications, or mode of delivery. However, they averaged 7.62 points higher on the Center for Epidemiological Studies Depression Scale (CES-D) $(p=<0.01)$. In addition, 20 women did not complete the CESD, and 35 women did not complete the Tolerance, Worried, Eye-opener, Amnesia, and K/Cut down (TWEAK) alcohol screener. The analyses reported here included only the women who had nonmissing values for all

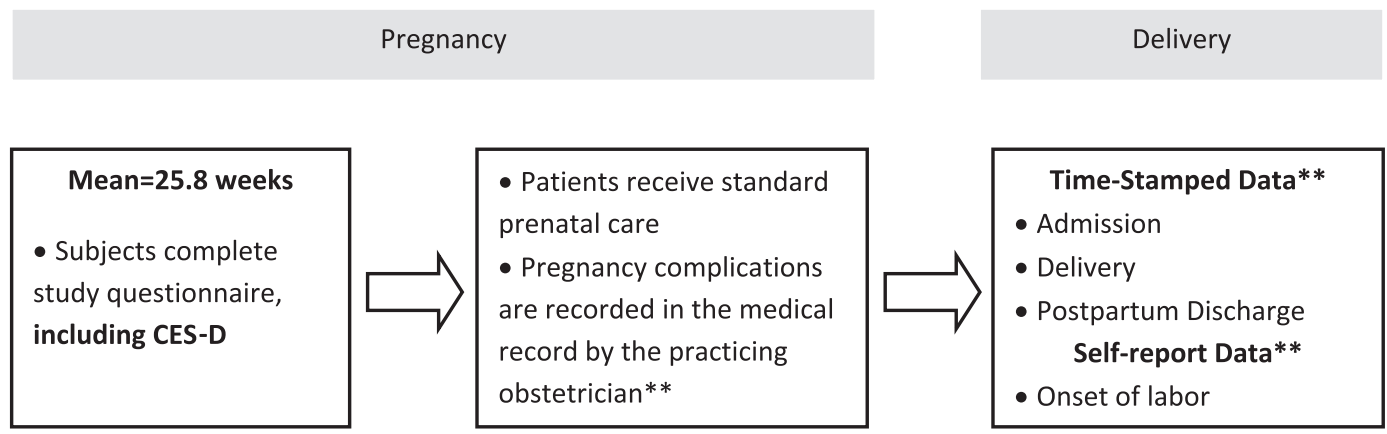

FIG. 1. Project timeline. CES-D, Center for Epidemiological Studies Depression Scale. ${ }^{* *}$ Data collected from the medical record. 


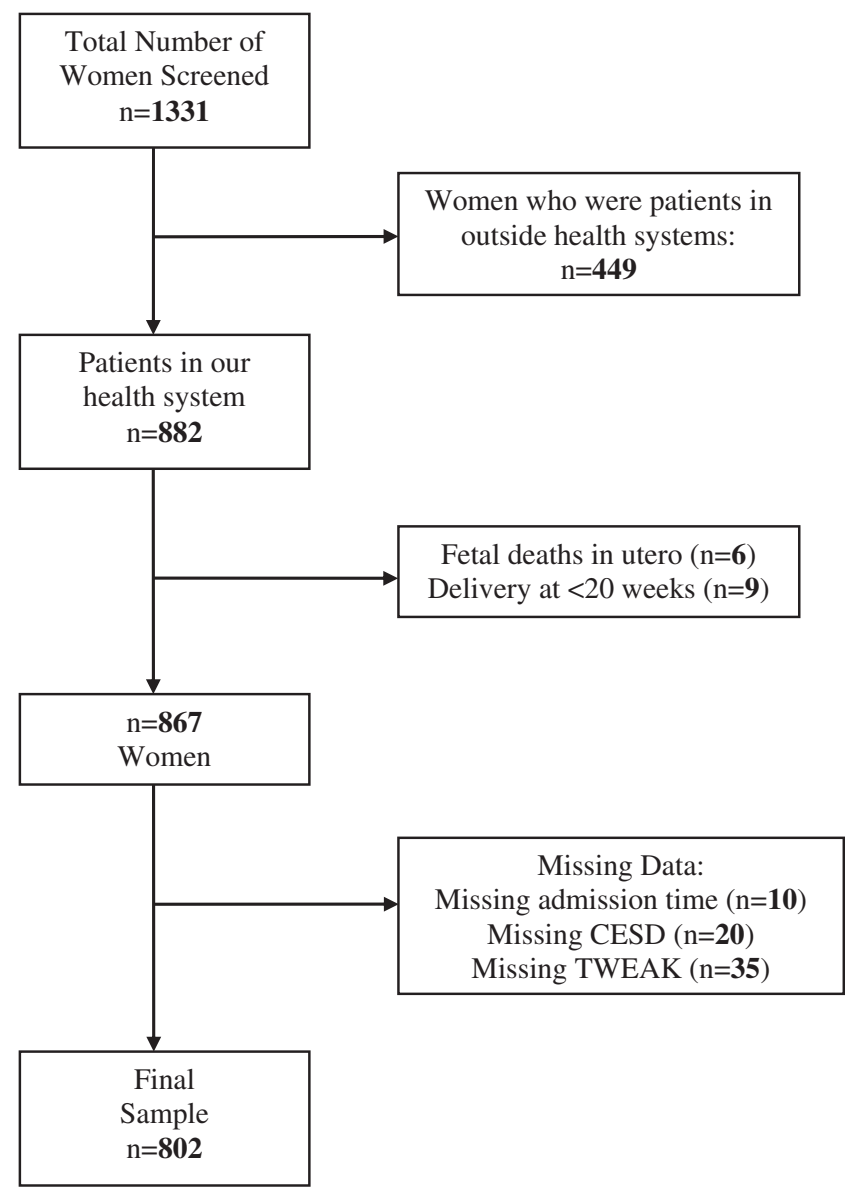

FIG. 2. Recruitment and study sample. TWEAK, Tolerance, Worried, Eye-opener, Amnesia, and K/Cut down, alcohol screener.

relevant time of event data and covariates ( $n=802$ of 867 eligible women, 92.5\%) (Fig. 2).

\section{Survey instrument and clinical data}

Predictor variables. General survey measures included demographic information, ratings of overall health, selfreported smoking during pregnancy, and use of prescription medications. In addition, past history of depression was measured using items derived from the Diagnostic Interview Schedule (DIS-III-R). ${ }^{28}$ These items ask participants if "you had two weeks or more when nearly every day you felt sad, blue, or depressed or in which you lost all interest in things like work?" The sensitivity of these items as screeners for depression or dysthymia within the last year ranges from 0.83 to $0.94 .^{29}$

Current depressive symptoms. Current depressive symptomatology was measured by the CES-D. ${ }^{30}$ Items on the CES-D cover the previous 7 days and are rated on a 4-point scale. A total score is derived by summing the ratings across the scale's 20 items. The CES-D shows good internal consistency in the general population (Cronbach's alpha $=0.84) .{ }^{31} \mathrm{In}$ a postpartum sample, the sensitivity and specificity of the CES-D to detect depression (major and minor) were 0.60 and 0.92 , respectively. ${ }^{32}$ This is comparable to the diagnostic properties of other depression screening instruments in the perinatal population. ${ }^{1}$ We used the standard cutoff point of $\geq 16$ to determine elevated depressive symptoms. ${ }^{33,34} \mathrm{We}$ chose a cutoff of 16 because in the original article using the CES-D in the general population, a cutoff of $\geq 16$ discriminated well between a general population sample and a psychiatric inpatient sample. ${ }^{30}$ Since that time, $\geq 16$ has been used as a recommendation for determining clinically significant symptoms. ${ }^{35}$ In addition, a cutoff of $\geq 16$ has been used in screening studies for perinatal depression. ${ }^{1}$

Problem alcohol use. Problem alcohol use was assessed with the TWEAK alcohol screener. The TWEAK is a 5-item screener that contains questions from the Michigan Alcohol Screening Test (MAST), Cut down, Annoyed, Guilty and Eyeopener (CAGE), and Tolerance, Annoyed, Cut down, Eyeopener (T-ACE) ${ }^{36}$ It yields a total severity score of $0-7$. Using a cutoff score of 2 , the TWEAK demonstrates good sensitivity and specificity in screening for at-risk drinking in pregnant women. ${ }^{37}$ In the HPP study, women with a positive alcohol screener or an elevated CES-D received pschyoeducational materials in the mail. Patients who screened positive for depression received information about the signs and symptoms of depression and referral information. Patients who screened positive for problem alcohol use received a packet of information about the effects of alcohol use during pregnancy, including information on fetal alcohol syndrome, and referral information.

Additional predictor variables. Additional questions evaluated the use of depression medications in the 2 years before the study. The survey also assessed whether women were receiving counseling, psychotherapy, or medication for emotional problems at the time of screening. In addition, we reviewed the electronic medical record to extract breast vs. bottle feeding status, obstetric complications (including antepartum, intrapartum, and postpartum complications), mode of delivery, anesthesia method for delivery, patient selfreport of the time of labor onset, and infant birth weight and Apgar scores. Antepartum complications included maternal complications of pregnancy, such as preeclampsia, preterm labor, and gestational diabetes; fetal complications of pregnancy, such as major fetal anomalies and intrauterine growth restriction; and medical comorbidities during pregnancy, such as chronic renal insufficiency and chronic hypertension. Intrapartum complications included nonreassuring fetal heart rate tracing necessitating intervention, chorioamnionitis, intrapartum abruption, malpresentation, shoulder dystocia, fourth degree laceration, arrest of labor, and arrest of descent. Postpartum complications included retained placenta, postpartum hemorrhage, postpartum deep venous thrombosis or pulmonary embolus, postpartum wound infection, postpartum endometritis, and mastitis. Because antepartum complications were common in our sample and some women experienced more than one antepartum complication, we created a composite variable for antepartum complications, representing the total number of complications for each subject.

We also calculated a modified Kotelchuck Adequacy of Prenatal Care Utilization Index. ${ }^{38}$ The Kotelchuck Index uses two data points to categorize the adequacy of prenatal care. First, the time of initiation of prenatal care is recorded. Second, the number of prenatal care visits attended is compared with the expected number of visits for the period between the 
initiation of care and delivery, based on prenatal care standards of the American College of Obstetricians and Gynecologists (ACOG). Finally, the Index combines these two data points to create a single summary score of adequate plus, adequate, intermediate, or inadequate. Because some of our study participants were referred for care, we did not have complete data for the timing of the first prenatal visit. Therefore, we calculated prenatal care adequacy based on the number of weeks from the time of presentation to the study health system. We categorized women by the percentage of recommended visits attended during this interval, using the cutoffs of the original Kotelchuck Index (inadequate, $<50 \%$ of expected visits; intermediate, 50\%-79\%; adequate, $80 \%-$ $109 \%$; adequate plus, $>109 \%$ ). We also obtained data from accompanying demographic records about insurance status of the mother and the child. We collected hospital costs associated with the peripartum stay from our hospital billing database.

Outcome variables. To measure our outcomes, we collected length of stay data for each subject from the hospitalization in which delivery occurred. In our previous work with this patient sample ${ }^{8}$, we compared peripartum length of stay using available hospital claims data from our health network. However, peripartum length of stay was recorded in aggregate for billing purposes, in the number of whole days between obstetric admission and postpartum discharge. This form of data collection negated the possibility of studying predelivery vs. postdelivery phenomena. Given this limitation, we searched for different data collection systems after our prior study and identified our electronic medical record charting system as a potential data source. From the charting system, we were able to extract date and time-stamped data for each woman in the sample, including the time of admission, time of delivery, and time of postpartum discharge. We also collected time of labor onset from the electronic medical record. This was not a time-stamped event but was instead a self-report of the onset of labor collected during the admission history and physical examination.

The primary outcomes of interest for this project were predelivery and postdelivery length of stay. We used the chart-recorded dates and times of admission, delivery, and discharge to calculate three forms of length of stay for our analyses: (1) total peripartum stay, the time from admission to the obstetrics unit to postpartum discharge, (2) predelivery stay, the time from admission to delivery of the infant, and (3) postdelivery stay, the time from delivery until postpartum discharge.

\section{Statistical analysis}

We used descriptive statistics to report patient characteristics and obstetric outcomes for the study population. In addition, we used $t$ tests and chi-square analyses to compare sociodemographic factors, substance abuse, and obstetric outcomes between women with and without elevated depressive symptoms during pregnancy, and we used a $t$ test for samples with unequal variances to compare hospital charges between these same subgroups.

Because of the skewed distribution of our length of stay outcomes, we used a time-to-event analysis to compare length of stay. We used nonparametric Kaplan-Meier curves and the log-rank test in bivariate analyses to compare total peripartum, predelivery, and postdelivery stays between women with and without depressive symptoms during pregnancy. We used a semiparametric Cox proportional hazards regression to examine potential predictors of length of stay. We used our conceptual model, based on review of the literature on peripartum length of stay, to determine which independent variables to test for inclusion into the Cox regression: parity, antepartum complications, induction of labor, gestational age, multiple pregnancy, sociodemographics, problem drinking, smoking status, current depression treatment, mode of delivery, mode of anesthesia, rupture of membranes (artificial vs. spontaneous), birth weight, insurance status, prenatal care adequacy, breastfeeding status, neonatal length of stay, and postpartum complications. Variables with a bivariate association with length of stay at $p<0.2$ were included in the final Cox model.

Finally, we tested our model for violations of the proportional hazards assumption using the link test and the test of Schoenfeld residuals. We tested the model for goodness of fit using Cox-Snell residuals. The final regression model displayed excellent fit. We also examined residuals for influential outliers. One influential outlier was noted. We ran our regression model with and without this case, and there was no significant difference in outcomes (in fact, depression's effect on length of stay was stronger in the model with the outlier removed).

To test the interaction between depressive symptoms and parity, we performed a stratified log-rank test for equality of survivor functions. We also ran stratified Cox regression models by parity and compared the hazard ratio for depression between the two models. All analyses were conducted with STATA ${ }^{\circledR} 10$ (StataCorp, College Station, TX). A $p$ value of $<0.05$ was considered statistically significant.

\section{Results \\ Description of study sample}

Participant characteristics $(n=802)$ are shown in Table 1. The average maternal age at study entry was 29.4 years (SD 5.5). Most women were Caucasian, and the majority had private health insurance. Approximately $90 \%$ of the women were married or cohabiting. Over one half of the women in the study graduated from high school. More women reported alcohol use during pregnancy $(16.3 \%)$ than cigarette smoking $(12.6 \%)$. Most mothers rated adequate or adequate plus on a modified Kotelchuck Index (78.1\%). Almost one quarter of women experienced at least one antepartum complication $(21.4 \%)$, which included hypertensive disorders, gestational diabetes, preterm labor, preterm delivery, preterm premature rupture of membranes (PPROM), major fetal anomalies, maternal autoimmune disorder, intrauterine growth restriction, and incompetent cervix.

Delivery outcomes are displayed in Table 2. The mean gestational age at delivery was 38.8 weeks (SD 2.4); $27.4 \%$ of women received a cesarean delivery $(n=220)$, and $40.9 \%$ of these 220 deliveries were scheduled, elective cesareans $(59.1 \%$ were indicated cesarean deliveries after admission). Only $9.6 \%$ of mothers experienced a postpartum complication. The majority $(79.4 \%)$ of mothers breastfed their infants.

Overall, $18.4 \%$ of mothers scored above the cutoff for depressive symptomatology on the CES-D $(n=148)$. Approximately 
Table 1. Subject Characteristics

\begin{tabular}{|c|c|c|c|c|}
\hline & Total $(\mathrm{n}=802)$ & $C E S-D \geq 16(\mathrm{n}=148)$ & $C E S D<16(\mathrm{n}=654)$ & $\mathrm{p}$ \\
\hline $\operatorname{Age}^{a}$ & $29.4(5.5)$ & $28.2(5.5)$ & $29.7(5.5)$ & $<0.01$ \\
\hline \multicolumn{5}{|l|}{ Race } \\
\hline Non-Hispanic white & $630(78.5)$ & 115 (77.7) & $515(78.7)$ & 0.78 \\
\hline Married/cohabiting & $726(90.5)$ & $114(77.0)$ & $612(93.6)$ & $<0.01$ \\
\hline \multicolumn{5}{|l|}{ Education } \\
\hline$\leq$ High school & $173(21.6)$ & $51(34.5)$ & $122(18.6)$ & \multirow[t]{3}{*}{$<0.01$} \\
\hline Some college/college graduate & $374(46.6)$ & $69(46.6)$ & $305(46.6)$ & \\
\hline Beyond college & $255(31.8)$ & $28(18.9)$ & $227(34.7)$ & \\
\hline \multicolumn{5}{|l|}{ Employment } \\
\hline Unemployed & $306(38.1)$ & $77(52.0)$ & $229(35.0)$ & \multirow[t]{3}{*}{$<0.01$} \\
\hline Part-time & $143(17.8)$ & $21(14.2)$ & $122(18.6)$ & \\
\hline Full-time & $353(44.0)$ & $50(33.8)$ & $303(46.3)$ & \\
\hline \multicolumn{5}{|l|}{ Insurance status } \\
\hline Public (Medicare/Medicaid) & $50(6.2)$ & $21(14.2)$ & $29(4.4)$ & \multirow[t]{2}{*}{$<0.01$} \\
\hline Private & $752(93.8)$ & $127(85.8)$ & $625(95.6)$ & \\
\hline Multiparous & $422(52.6)$ & $84(56.8)$ & $338(51.7)$ & 0.26 \\
\hline Smoker & $101(12.6)$ & $37(25.0)$ & $64(9.8)$ & $<0.01$ \\
\hline TWEAK $\geq 2$ & $76(9.5)$ & $24(16.2)$ & $52(7.9)$ & $<0.01$ \\
\hline Lifetime history of depression & $253(31.5)$ & $93(62.8)$ & $160(24.5)$ & $<0.01$ \\
\hline Current depression treatment & $50(6.2)$ & $28(18.9)$ & $22(3.4)$ & $<0.01$ \\
\hline \multicolumn{5}{|l|}{ Kotelchuck Index, modified } \\
\hline Inadequate & $44(5.5)$ & $13(8.8)$ & $31(4.7)$ & \multirow[t]{4}{*}{0.13} \\
\hline Intermediate & $240(29.9)$ & $41(27.7)$ & $199(30.4)$ & \\
\hline Adequate & $401(50.0)$ & $68(45.9)$ & $333(50.9)$ & \\
\hline Adequate plus & $117(14.6)$ & $26(17.6)$ & $91(13.9)$ & \\
\hline \multicolumn{5}{|l|}{ Antepartum complications } \\
\hline Preeclampsia or PIH & $37(4.6)$ & $5(3.4)$ & $32(4.9)$ & 0.43 \\
\hline Chronic hypertension & $12(1.5)$ & $4(2.7)$ & $8(1.2)$ & 0.18 \\
\hline Gestational diabetes & $36(4.5)$ & $5(3.4)$ & $31(4.7)$ & 0.47 \\
\hline Preterm labor & $23(2.9)$ & $9(6.1)$ & $14(2.1)$ & $<0.01$ \\
\hline PPROM & $18(2.2)$ & $5(3.4)$ & $13(2.0)$ & 0.30 \\
\hline Fetal anomalies & $12(1.5)$ & $2(1.3)$ & $10(1.5)$ & 0.87 \\
\hline Maternal autoimmune disorder & $8(1.0)$ & $2(1.3)$ & $6(0.9)$ & 0.63 \\
\hline IUGR & $6(0.75)$ & $0(0)$ & $6(0.9)$ & 0.24 \\
\hline Incompetent cervix & $20(2.5)$ & $4(2.7)$ & $16(2.4)$ & 0.86 \\
\hline Multiple pregnancy & $42(5.2)$ & $8(5.4)$ & $34(5.2)$ & 0.92 \\
\hline
\end{tabular}

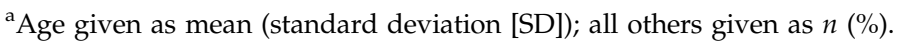

CES-D, Center for Epidemiological Studies Depression Scale; IUGR, intrauterine growth retardation; PIH, pregnancy-induced hypertension; PPROM, preterm premature rupture of membranes; TWEAK, Tolerance, Worried, Eye-opener, Amnesia, K/Cut down.

$19 \%$ of the women with a positive CES-D reported any current form of treatment $(n=28)$, and almost two thirds of the women with a positive CES-D had a prior history of depression in their lifetime $(n=93)$. In addition, $3.4 \%$ of women with a CES-D $<16$ reported current treatment for depression, likely reflecting women who had improved symptomatology on their current treatment regimen.

In bivariate analyses, mothers with elevated depressive symptoms were younger and less educated than women without elevated depressive symptoms. They were more likely to be single, unemployed, and publicly insured (Table 1). One quarter of mothers with elevated depressive symptoms reported smoking during the index pregnancy, and they were twice as likely to have an elevated TWEAK compared to women without elevated depressive symptoms. Women with elevated depressive symptoms did not differ by the rate of labor induction, type of labor anesthesia, or mode of delivery (Table 2), including the type of cesarean delivery. However, women with elevated depressive symptoms were almost three times as likely to experience spontaneous preterm labor (onset of labor at $<37$ weeks' gestation) (Table 1) and over twice as likely to deliver at $\leq 34$ weeks' gestation (Table 2), including both spontaneous preterm deliveries and indicated preterm deliveries because of high obstetric risk.

In our study population, the mean hospital charges for a maternal peripartum stay equaled $\$ 5,665$ (SD $\pm \$ 2,201)$, and the mean charges were about $8 \%$ higher in women with an elevated CES-D ( $\$ 461, p=0.05)$.

\section{Length of stay outcomes}

The total mean peripartum stay (admission to discharge) was 54.7 hours (SD \pm 28.6 ), with mean predelivery (admission to delivery) and postdelivery (delivery to discharge) stays of 13.6 hours ( $\mathrm{SD} \pm 23.3$ ) and 41.5 hours ( $\mathrm{SD} \pm 17.3$ ), respectively. Women with elevated depressive symptoms were significantly more likely to have longer total peripartum stays $(p<0.001)$. In bivariate analysis, there was no significant difference in postdelivery stay between women with and without an elevated CES-D (log-rank test chi-square $(1)=0.53$, $p=0.46)$. However, women with an elevated CES-D showed a significant increase in predelivery stay (log-rank test 
Table 2. Delivery Outcomes

\begin{tabular}{|c|c|c|c|c|}
\hline & Total $(\mathrm{n}=802)$ & $C E S D \geq 16(\mathrm{n}=148)$ & $C E S D<16(\mathrm{n}=654)$ & $\mathrm{p}$ \\
\hline Gestational age at delivery (weeks) & $38.8(2.4)$ & $38.6(2.5)$ & $38.9(2.4)$ & 0.19 \\
\hline Delivery $\leq 34$ weeks gestation, $n(\%)$ & $34(4.2)$ & 11 (7.4) & $23(3.5)$ & 0.03 \\
\hline \multicolumn{5}{|l|}{ Mode of delivery, $n(\%)$} \\
\hline Spontaneous vaginal & $410(51.1)$ & 69 (46.6) & 341 (52.1) & \multirow[t]{3}{*}{0.48} \\
\hline Assisted vaginal & $172(21.4)$ & $35(23.6)$ & $137(20.9)$ & \\
\hline Cesarean & $220(27.4)$ & $44(29.7)$ & $176(26.9)$ & \\
\hline \multicolumn{5}{|l|}{ Anesthesia, $n(\%)$} \\
\hline None & $45(5.6)$ & $8(5.4)$ & $37(5.7)$ & \multirow[t]{4}{*}{0.13} \\
\hline Local & $159(19.8)$ & $20(13.5)$ & $139(21.2)$ & \\
\hline Regional & $581(72.4)$ & $115(77.7)$ & $466(71.2)$ & \\
\hline General & $17(2.1)$ & $5(3.4)$ & $12(1.8)$ & \\
\hline Induction of labor, $n(\%)$ & $124(15.4)$ & $23(15.5)$ & $101(15.5)$ & 0.70 \\
\hline Birth weight $(\mathrm{kg})$ & $3.4(0.6)$ & $3.3(0.7)$ & $3.4(0.6)$ & 0.32 \\
\hline Postpartum complications, $n(\%)$ & $77(9.6)$ & $14(9.5)$ & $63(9.6)$ & 0.95 \\
\hline Breastfeeding, $n(\%)$ & $637(79.4)$ & $113(76.3)$ & $524(80.1)$ & 0.30 \\
\hline Hospital charges, in thousands & $5.7(2.2)$ & $6.0(3.3)$ & $5.6(1.9)$ & 0.02 \\
\hline
\end{tabular}

Results presented as mean (SD) unless otherwise indicated.

chi-square $(1)=4.54, p=0.03$ ) (Fig. 3). Although this effect was most pronounced at the far right of the distribution, the difference between depressed and nondepressed women was apparent starting at approximately 12 hours of stay.

Each of the following variables was also significantly associated with a longer predelivery stay: antepartum complications, induction of labor, full-time employment, TWEAK $\geq 2$, and general anesthesia (all $p$ values $\leq 0.01$ except general anesthesia, $p=0.03$ ). There was a trend toward a longer predelivery stay in women receiving a cesarean delivery $(p=0.08)$. On the other hand, a shorter predelivery stay was significantly associated with increasing gestational age, increasing maternal age, greater than a college education, and multiparity (all $p$ values $\leq 0.01$ except greater than college education, $p=0.02$ ). Receiving treatment for depression was not significantly related to predelivery stay in bivariate analyses $(p=0.10)$.

We also discovered a significant interaction effect between depressive symptoms and parity. In multiparous patients, an elevated CES-D was significantly associated with longer predelivery stay, but in primiparous patients, elevated depressive symptoms did not significantly impact predelivery length of stay (stratified log-rank test for equality of survivor functions chi-square $(1)=6.62, p=0.01$ ). Overall, multiparous women were significantly less likely than primiparous women to have antepartum complications ( $24.7 \%$ vs. $33.8 \%$, respectively) and significantly more likely to have a shorter predelivery stay (mean 10.2 hours vs. 17.4 hours, respectively). When compared to nondepressed multiparas, however, multiparous women with an elevated CES-D showed a greater increase in both length of stay (multiparas with CES$\mathrm{D}<16$ : 8.4 hours vs. multiparas with CES-D $\geq 16$ : 17.9 hours) and antepartum complications (multiparas with CES-D $<16$ : $23.7 \%$ vs. multiparas with CESD $\geq 16: 30.8 \%$ ) than primiparas under the same comparisons (primiparas antepartum complication rate: $C E S-D<16: 33.0 \%$ vs. CES-D $\geq 1633.8 \%$; mean length of stay: CESD <16: 16.9 hours vs. CES-D $\geq 16: 20.8$ hours) (Fig. 4). Multiparous and primiparous subjects did not differ significantly in the frequency of CES-D scores $\geq 16$ (84, $19.9 \%$, vs. $64,16.8 \%, p=0.26)$. In addition, the two groups did not differ in the number of women receiving depression treatment $(28,6.7 \%$, vs. $22,5.8 \%, p=0.60)$.

Because of the interaction between parity and depressive symptoms, we stratified our multivariate regression models by parity (Table 3 ). Also, we present only the results of our multivariate model for predelivery stay because depressive symptoms were not significantly related to postdelivery stay in bivariate analysis. As noted, there was no significant association between predelivery stay and depressive symptoms in primiparous subjects. Table 3 demonstrates that in multiparous subjects, an CES-D < 16 was significantly related to a greater odds of delivery at any point from the time of admission, meaning that women without elevated depressive symptoms were significantly more likely to have shorter predelivery stays (they were more likely to deliver at an earlier point). Therefore, in multiparous subjects, an elevated CES-D was significantly related to a longer predelivery stay. This effect was partially influenced by sociodemographic factors, but it remained significant until antepartum complications were added to the model (Table 3). Birth weight, mode of delivery, depression treatment, and problem drinking (TWEAK score) did not significantly influence the association between depressive symptoms during pregnancy and increased predelivery stay.

Finally, we wanted to determine if the increase in predelivery stay was due to antepartum or intrapartum effects. Using a patient self-report variable of the timing of labor onset, we divided predelivery stay into antepartum stay, the time from admission to the onset of labor, and intrapartum stay, the time from onset of labor to delivery. There was no significant difference in intrapartum stay between women with and without elevated depressive symptoms. However, an elevated depression screen during the pregnancy was significantly associated with a $140 \%$ increase in antepartum stays of $\geq 24$ hours, which represent stays at $\geq 95$ th percentile (Fig. 5).

We also controlled for additional obstetric variables in our Cox regression model, including induction of labor, artificial rupture of membranes, and type of anesthesia. These variables did not significantly alter the results, likely because the 

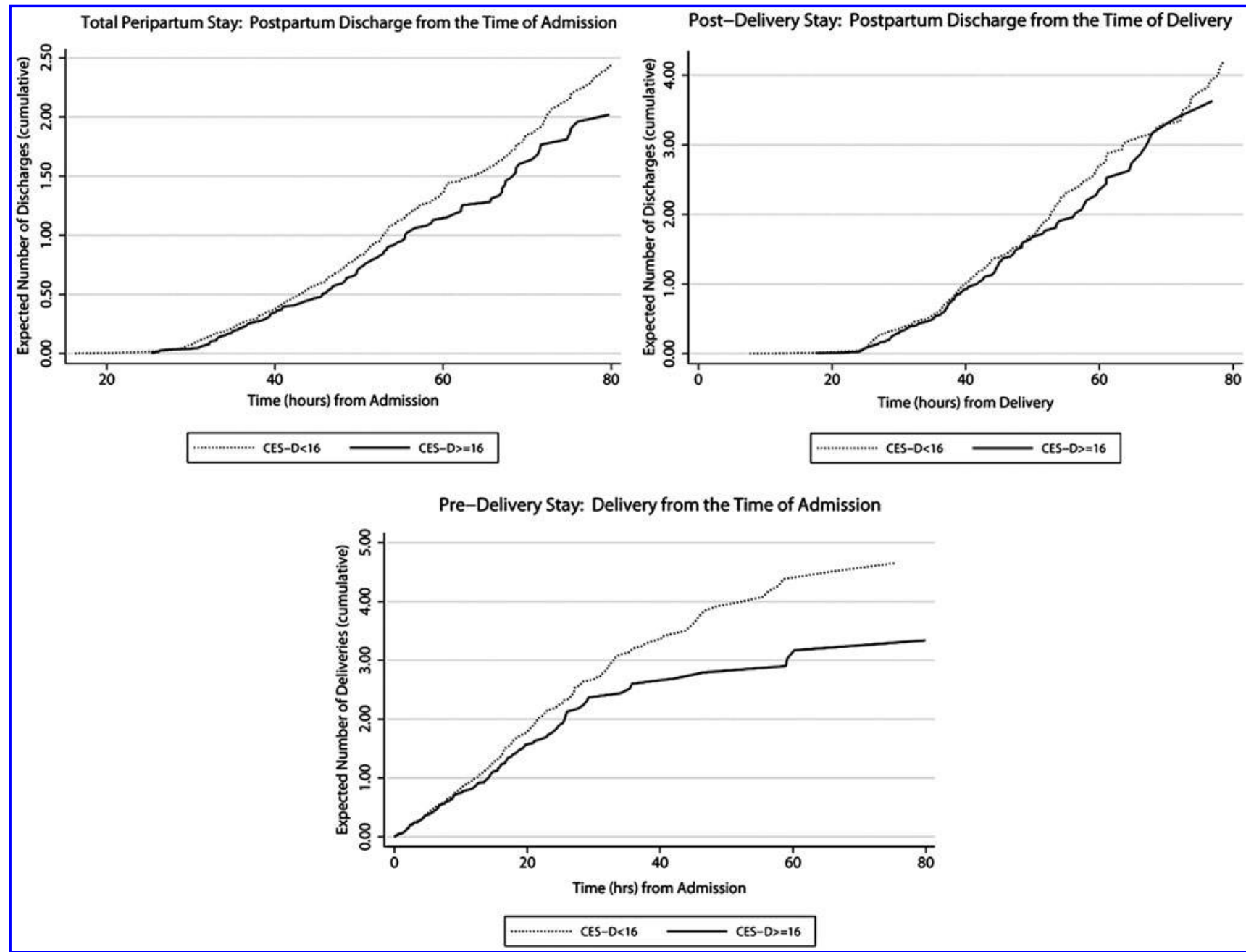

FIG. 3. Total, postdelivery, and predelivery stay by depressive symptom status. Expected number of deliveries and expected number of discharges (cumulative) represent the number of times we would mathematically expect a woman to have delivered or been discharged, respectively, over the given period of time if only delivery and discharges were repeatable. ${ }^{39}$
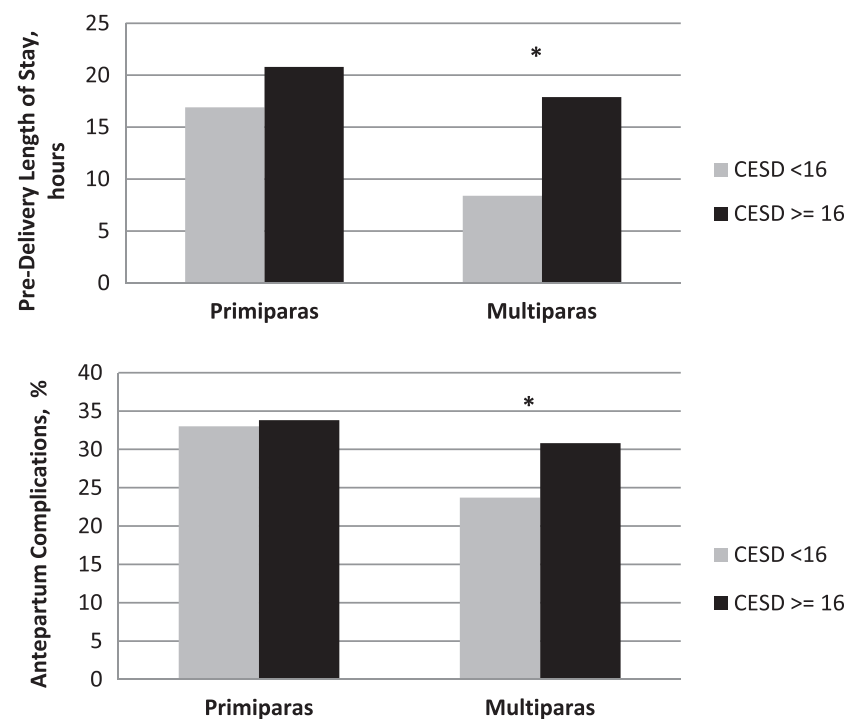

FIG. 4. Predelivery length of stay and antepartum complications, by party and depressive symptom status. Black bars represent women with a CES-D $\geq 16$; gray bars represent women with a CES-D $<16 .{ }^{*} p<0.05$. difference in stay appeared to be antepartum rather than intrapartum. In addition, induction of labor failed to meet the proportional hazards assumption and was not included in the final model.

\section{Discussion}

The key finding of this study is that depressive symptoms during pregnancy are significantly associated with longer predelivery stays at the end of pregnancy, and this relationship between antepartum depressive symptoms and predelivery stay is mediated in part by antepartum complications, even after controlling for sociodemographic factors. In this study, women with elevated depressive symptoms were significantly more likely to have antepartum stays at or above the 95th percentile. This increase in extensive antepartum stays resonates with the findings of our Cox regression because antepartum complications are often associated with such long hospital stays, and antepartum complications mediated in part the relationship between depressive symptoms and predelivery length of stay.

These findings refine our previous work, which has shown that depressive symptoms are significantly associated with an 
Table 3. Multivariate Model: Predelivery Length of Stay, Multiparous Patients

\begin{tabular}{|c|c|c|c|c|c|}
\hline \multirow[b]{2}{*}{ Patient characteristics } & \multicolumn{5}{|c|}{ Hazard ratio: Odds of delivery from time of admission } \\
\hline & Model A & Model B & Model C & Model D & Model E \\
\hline$C E S-D<16$, nondepressed $^{\mathrm{a}}$ & $1.37(1.08-1.74)$ & $1.30(1.02-1.65)$ & $1.23(0.97-1.57)$ & $1.24(0.97-1.58)$ & $1.21(0.93-1.58)$ \\
\hline \multicolumn{6}{|l|}{ Demographic confounders } \\
\hline Maternal Age & & $1.00(0.98-1.02)$ & $1.00(0.98-1.02)$ & $1.00(0.98-1.02)$ & $1.00(0.98-1.02)$ \\
\hline \multicolumn{6}{|c|}{ 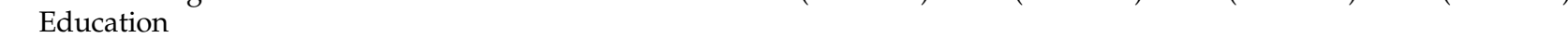 } \\
\hline$\leq$ High school & & Reference & Reference & Reference & Reference \\
\hline Some college/college graduate & & $1.09(0.86-1.38)$ & $1.09(0.86-1.38)$ & $1.09(0.86-1.38)$ & $1.14(0.89-1.46)$ \\
\hline Beyond college & & $1.49(1.11-2.00)$ & $1.43(1.06-1.91)$ & $1.37(1.02-1.84)$ & $1.34(0.98-1.82)$ \\
\hline \multicolumn{6}{|l|}{ Employment } \\
\hline Unemployed & & Reference & Reference & Reference & Reference \\
\hline Part-time employment & & $1.17(0.91-1.51)$ & $1.17(0.91-1.50)$ & $1.19(0.93-1.53)$ & $1.20(0.92-1.55)$ \\
\hline Full-time employment & & $0.92(0.74-1.15)$ & $0.93(0.75-1.16)$ & $0.97(0.77-1.21)$ & $1.03(0.81-1.30)$ \\
\hline Antepartum complications, composite & & & $0.67(0.56-0.80)$ & $0.72(0.60-0.86)$ & $0.64(0.52-0.78)$ \\
\hline Multiple pregnancy & & & & $1.09(0.68-1.73)$ & $1.01(0.61-1.66)$ \\
\hline Birth weight & & & & $1.19(1.03-1.39)$ & $1.13(0.97-1.32)$ \\
\hline \multicolumn{6}{|l|}{ Mode of delivery } \\
\hline Spontaneous vaginal & & & & Reference & Reference \\
\hline Assisted vaginal & & & & $0.96(0.76-1.21)$ & $0.97(0.76-1.24)$ \\
\hline Cesarean section & & & & $1.07(0.85-1.36)$ & $1.14(0.89-1.46)$ \\
\hline Current depression treatment & & & & & $1.13(0.75-1.70)$ \\
\hline TWEAK $\geq 2$ & & & & & $0.85(0.60-1.23)$ \\
\hline
\end{tabular}

aReference group: CES-D $\geq 16$.

increase in total peripartum stay. ${ }^{8}$ In our previous study, the effect of depression remained significant even after controlling for antepartum complications. This difference between the two studies is likely due to the fact that in this analysis we were able to separate models and, thus, the variance, before and after delivery. Indeed, in this model, antepartum complications did significantly influence the relationship between depressive symptoms and predelivery length of stay. In addition, we discovered a strong interaction between parity and depressive symptoms. We are unable to explain why this interaction exists, but perhaps there is an additive effect between the stress of having other children at home (which may be accompanied by greater financial and family stress) and depressive symptoms during the pregnancy.

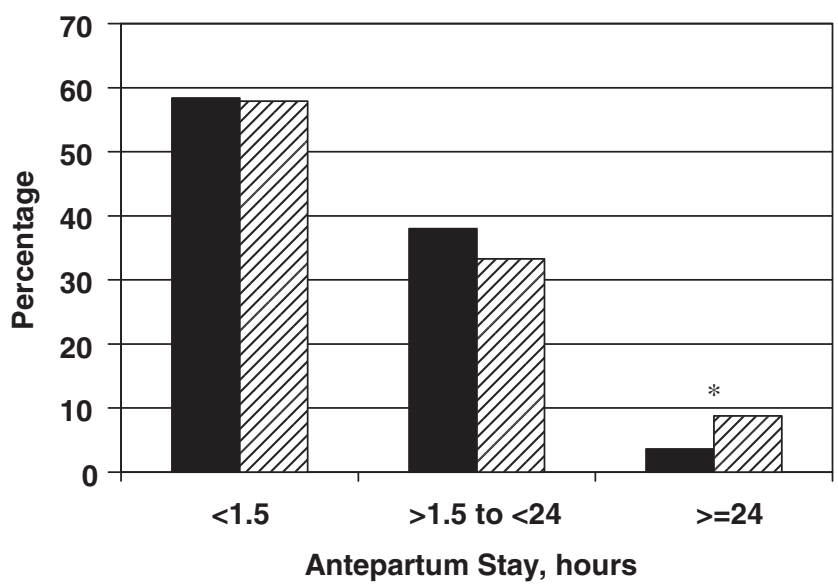

FIG. 5. Antepartum stay by depression status. Solid bars represent women with CES-D <16; striped bars represent women with CES-D $\geq 16$. ${ }^{*} p<0.05$.
We were also surprised that current depression treatment did not significantly affect maternal length of stay. The variable for depression treatment included medication, psychotherapy, or counseling of any kind. Our power to detect a treatment effect was lowered by the small percentage of women receiving depression treatment (6.6\% of the total sample). In addition, women receiving depression treatment had significantly higher CES-D scores. This may reflect the severity of disease in women receiving treatment, but it may also indicate that women were not receiving adequate therapy for their disease, which has been demonstrated as a common problem in the perinatal population. ${ }^{40}$ Because of these limitations, we cannot determine if effective depression treatment can influence the relationship between depressive symptoms and maternal length of stay. Future treatment trials should investigate the potential of effective treatments to change obstetric outcomes.

Our study has several limitations. First, we relied on a selfreport screener for depression instead of a diagnostic interview. However, research findings have linked symptom measures to poor pregnancy and birth outcomes. ${ }^{41,} 42$ Nonetheless, elevated symptoms on a depression screen, such as the CES-D, could be associated with psychiatric illness other than depression, especially anxiety. Also, the two DIS-III-R items used to screen for past history of depression have not been validated within the setting of pregnancy. Second, our findings may not generalize to other clinical populations, as our study population was relatively homogeneous and of high obstetric risk (almost one fourth of the sample had an antepartum complication). It is important to examine the applicability of our findings to other populations, particularly low-income, minority women. Because of our cohort study design, we cannot ensure that we controlled for all potential confounders, and although our multivariate results suggest a causal pathway, this cannot be confirmed. Although the 
majority of the antepartum complications in our sample had onset after the depression screen was completed, this may not have been the case with all the complications. In addition, the effect of depression was greatest at the extreme of the length of stay distribution, where the sample size is limited. However, depression's effect on length of stay was apparent in our sample as early as 12 hours from the time of admission. Finally, we used a self-report measure for the timing of labor onset.

Our bivariate analysis demonstrated that women with elevated depressive symptoms are significantly more likely to deliver at $<34$ weeks' gestation. Other studies have also demonstrated a relationship between antepartum depressive symptoms and preterm delivery. ${ }^{11,12,43}$ Studies have varied in whether the outcome measured was spontaneous preterm birth (birth secondary to spontaneous labor or PPROM) vs. premature birth due to any cause. In this study, a composite measure of antepartum complications, including preterm labor, PPROM, hypertensive disorders, gestational diabetes, and other complications of pregnancy, influenced the relationship between depressive symptoms and longer predelivery hospital stays. We need future studies to examine potential mechanisms by which depression may be related to such complications.

Although the adverse consequences of antepartum complications may be self-explanatory, prolonged maternal hospitalizations also have several potential negative effects. For the system, prolonged predelivery hospitalizations may lead to a backup of patients on inpatient high-risk antepartum wards. In addition, as we have shown, these prolonged hospitalizations are related to a significant increase in hospital charges. ${ }^{8}$ For the patient, increased time in the hospital means time away from work and family. This may place added strain on other family members who must then take on the additional responsibilities that the mother maintained. Antepartum hospitalization (hospitalization before the onset of labor for an antepartum complication, such as pregnancyinduced hypertension) has also been associated with the development of elevated maternal depressive symptoms. ${ }^{44} \mathrm{We}$ do not know how the stress of hospitalization may affect women, like the $18 \%$ of women in our sample, who were already depressed before the time of admission.

In summary, our study demonstrates that depressive symptoms during pregnancy are significantly related to longer predelivery hospital stays, and antepartum complications in depressed women explain part of this phenomenon. We contribute to the existing literature by showing that depressive symptoms are associated with extensive antepartum hospitalizations, even in a sample of relatively high SES at a tertiary academic center. These findings further demonstrate a need for effective depression care during pregnancy. Future studies should evaluate whether interventions during pregnancy can reduce these adverse obstetric outcomes, including antepartum complications and extensive hospitalizations.

\section{Acknowledgments}

We acknowledge the Robert Wood Johnson Clinical Scholars Program for financial support and mentorship associated with this project and the Education Discovery Institute for financial support during the preparation of this article. We also acknowledge Ananda Sen, Ph.D., for his assistance with the statistical analysis.
A poster of this work was presented at The American College of Obstetricians and Gynecologists 57th Annual Clinical Meeting, May 2-6, 2009, and was awarded the Second Place Ephraim McDowell Poster Award, American College of Obstetricians and Gynecologists District V Annual District Meeting, October 17-19, 2008.

\section{Disclosure Statement}

No competing financial interests exist.

\section{References}

1. Gaynes BN, Gavin N, Meltzer-Brody S, et al. Perinatal depression: Prevalence, screening accuracy, and screening outcomes. Evidence report/technology assessment No. 119. (prepared by the RTI-University of North Carolina Evidence-based Practice Center, under contract No. 290-020016.) AHRQ Publication No. 05-E006-2. Rockville, MD: Agency for Healthcare Research and Quality, 2005.

2. Saravay SSM, Lavin MM. Psychiatric comorbidity and length of stay in the general hospital. A critical review of outcome studies. Psychosomatics 1994;35:233-252.

3. Holmes J, House A. Psychiatric illness predicts poor outcome after surgery for hip fracture: A prospective cohort study. Psychol Med 2000;30:921-929.

4. Hosaka T, Aoki T, Watanabe T, Okuyama T, Kurosawa H. Comorbidity of depression among physically ill patients and its effect on the length of hospital stay. Psychiatry Clin Neurosci 1999;53:491-495.

5. Verbosky LA, Franco KN, Zrull JP. The relationship between depression and length of stay in the general hospital patient. J Clin Psychiatry 1993;54:177-181.

6. Ng TP, Niti M, Tan WC, Cao Z, Ong KC, Eng P. Depressive symptoms and chronic obstructive pulmonary disease: Effect on mortality, hospital readmission, symptom burden, functional status, and quality of life. Arch Intern Med 2007; 167:60-67.

7. Oxlad M, Stubberfield J, Stuklis R, Edwards J, Wade TD. Psychological risk factors for increased post-operative length of hospital stay following coronary artery bypass graft surgery. I Behav Med 2006;29:179-190.

8. Lancaster C, Flynn H, Johnson TRB, Marcus S, Davis M. Peripartum length of stay for women with depressive symptoms during pregnancy. I Womens Health 2010;19: 31-37.

9. Centers for Disease Control and Prevention. National Vital Statistics System: Birth data. Available at www.cdc.gov/ nchs/births.htm Accessed February 18, 2010.

10. Kurki T, Hiilesmaa V, Raitasalo R, Mattila H, Ylikorkala O. Depression and anxiety in early pregnancy and risk for preeclampsia. Obstet Gynecol 2000;95:487-490.

11. Orr ST, James SA, Blackmore Prince C. Maternal prenatal depressive symptoms and spontaneous preterm births among African-American women in Baltimore, Maryland. Am J Epidemiol 2002;156:797-802.

12. Steer RA, Scholl TO, Hediger ML, Fischer RL. Self-reported depression and negative pregnancy outcomes. I Clin Epidemiol 1992;45:1093-1099.

13. Chambers CD, Johnson KA, Dick LM, Felix RJ, Jones KL. Birth outcomes in pregnant women taking fluoxetine. $\underline{N}$ Engl J Med 1996;335:1010-1015.

14. Costei AM, Kozer E, Ho T, Ito S, Koren G. Perinatal outcome following third trimester exposure to paroxetine. Arch Pediatr Adolesc Med 2002;156:1129-1132. 
15. Kallen B. Neonate characteristics after maternal use of antidepressants in late pregnancy. Arch Pediatr Adolesc Med 2004;158:312-316.

16. Moses-Kolko EL, Bogen D, Perel J, et al. Neonatal signs after late in utero exposure to serotonin reuptake inhibitors: Literature review and implications for clinical applications. JAMA 2005;293:2372-2383.

17. Zeskind PS, Stephens LE. Maternal selective serotonin reuptake inhibitor use during pregnancy and newborn neurobehavior. Pediatrics 2004;113:368-375.

18. Brown S, Lumley J. Reasons to stay, reasons to go: Results of an Australian population-based survey. Birth 1997;24:148-158.

19. Fiscella K, Meldrum S, Franks P. Post partum discharge against medical advice: Who leaves and does it matter? Matern Child Health J 2007;11:431-436.

20. Lichtenstein B, Brumfield C, Cliver S, Chapman V, Lenze D, Davis V. Giving birth, going home: Influences on when lowincome women leave hospital. Health (Lond) 2004;8:81-100.

21. Lof M, Svalenius EC, Persson EK. Factors that influence firsttime mothers' choice and experience of early discharge. Scand J Caring Sci 2006;20:323-330.

22. Margolis LH, Kotelchuck M. Midwives, physicians, and the timing of maternal postpartum discharge. J Nurse Midwifery 1996;41:29-35.

23. Margolis LH, Kotelchuck M, Chang HY. Factors associated with early maternal postpartum discharge from the hospital. Arch Pediatr Adolesc Med 1997;151:466-472.

24. Roberts SA, Kruger GB. Reasons for increased postnatal length of stay and women's perceptions of length of stay. Aust J Midwifery 2001;14:12-17.

25. Waldenstrom U, Aarts C. Duration of breastfeeding and breastfeeding problems in relation to length of postpartum stay: A longitudinal cohort study of a national Swedish sample. Acta Paediatr 2004;93:669-676.

26. Weiss M, Ryan P, Lokken L, Nelson M. Length of stay after vaginal birth: Sociodemographic and readiness-for-discharge factors. Birth 2004;31:93-101.

27. Weiss ME, Ryan P, Lokken L. Validity and reliability of the Perceived Readiness for Discharge After Birth Scale. J Obstet Gynecol Neonatal Nurs 2006;35:34-45.

28. Robins LLN, Helzer JJE, Croughan JJ, Ratcliff KKS. National Institute of Mental Health Diagnostic Interview Schedule. Its history, characteristics, and validity. Arch Gen Psychiatry 1981;38:381-389.

29. Rost K, Burnam MA, Smith GR. Development of screeners for depressive disorders and substance disorder history. Med Care 1993;31:189-200.

30. Radloff LS. The CES-D scale: A self-report depression scale for research in the general population. Appl Psychol Meas 1977;1:385-401.

31. Corcoran K, Fisher J. Measures for clinical practice: A sourcebook. New York: Free Press, 1987.
32. Campbell SB, Cohn JF. Prevalence and correlates of postpartum depression in first-time mothers. J Abnorm Psychol 1991;100:594-599.

33. Husaini BA. Depression in rural communities: Validating the CES-D scale. J Community Psychol 1980;8:20-27.

34. Myers JK, Weissman MM. Use of a self-report symptom scale to detect depression in a community sample. Am J Psychiatry 1980;137:1081-1084.

35. Rush AJ, First MB, Blacker D, eds. Handbook of psychiatric measures, 2nd ed. Arlington, VA: American Psychiatric Publishing, 2008.

36. Chang GG. Alcohol-screening instruments for pregnant women. Alcohol Res Health 2001;25:204-209.

37. Bradley KA, Boyd-Wickizer J, Powell SH, Burman ML. Alcohol screening questionnaires in women: A critical review. JAMA 1998;280:166-171.

38. Kotelchuck M. An evaluation of the Kessner Adequacy of Prenatal Care Index and a proposed Adequacy of Prenatal Care Utilization Index. Am J Public Health 1994;84:14141420.

39. Cleves MA, Gould WW, Gutierrez RG. An introduction to survival analysis using STATA, Revised ed. College Station, TX: Stata Press, 2004.

40. Flynn HA, Blow FC, Marcus SM. Rates and predictors of depression treatment among pregnant women in hospitalaffiliated obstetrics practices. Gen Hosp Psychiatry 2006;28: 289-295.

41. Jesse DE, Seaver W, Wallace D. Maternal psychosocial risks predict preterm birth in a group of women from Appalachia. Midwifery 2003;19:191-202.

42. Hoffman S, Hatch MC. Depressive symptomatology during pregnancy: Evidence for an association with decreased fetal growth in pregnancies of lower social class women. Health Psychol 2000;19:535-543.

43. Li D, Liu L, Odouli R. Presence of depressive symptoms during early pregnancy and the risk of preterm delivery: A prospective cohort study. Hum Reprod 2009;24:146-153.

44. Heaman M. Stressful life events, social support, and mood disturbance in hospitalized and non-hospitalized women with pregnancy-induced hypertension. Can J Nurs Res 1992; 24:23-37.

Address correspondence to: Christie L. Palladino, M.D., M.Sc.

Education Discovery Institute Georgia Health Sciences University 1120 15th Street, CJ 1020 Augusta, GA 30912

E-mail: cpalladino@georgiahealth.edu 